\title{
Marvelous Response of Severe Onychotillomania to Treatment with Local Steroid Injection plus Topical Calcipotriol/Betamethasone Dipropionate Combination
}

\author{
Hamed Mohamed Abdo \\ Department of Dermatology, Faculty of Medicine, Al Azhar University, Cairo, Egypt
}

\section{Established Facts}

- Onychotillomania is usually linked to emotional and psychologic factors, which vary greatly from mild anxiety to deep psychosis.

- Both onychotillomania and the underlying psychosis could exacerbate each other, setting up a vicious cycle.

- Onychotillomania remains a therapeutic challenge and a difficult management as it can be highly resistant to both dermatological and psychological interventions.

\section{Novel Insights}

- The current case represents complete resolution of the condition with local therapy, with complete restoration of the nail anatomy after 8 months.

- In parallel to treatment success, anxiety behavior diminished, and the patient did no longer feel the desire to injure her fingernail, breaking down the vicious cycle.

\section{Keywords}

Onychotillomania · Anxiety · Nail dystrophy · Local steroid injection - Topical betamethasone/dipropionate combination

\section{Abstract \\ Onychotillomania is a psychodermatosis that results from self-induced repeated trauma to the nail unit. It is character- ized by the neurotic and irresistible urge to pick at, pull out, or harmfully bite or injure the nail(s). Multiple psychological}

factors can be involved. It can be difficult to diagnose, as patients mostly deny the self-destructive behavior, and the clinical features may mimic other inflammatory conditions of the nail apparatus. It presents with typical, usually bizarre morphologies of the nail plate. There is no evidence-based treatment for the condition, and it can be highly resistant to both dermatological and psychological interventions. Based on history and classical nail changes, a case of onychotillomania in a 19-year-old woman successfully treated with local steroid injection plus topical calcipotriol/betamethasone dipropionate combination is presented. An injection of a local 
steroid ( $0.2 \mathrm{~mL}$ of $5 \mathrm{mg} / \mathrm{mL}$ triamcinolone acetonide) was given monthly for 3 months and was then decreased to a bimonthly interval. In addition, topical calcipotriol/betamethasone dipropionate combination was applied daily for 3 months and was then decreased to every other day. Clinical improvement was noted after the third month, while complete resolution of the condition was achieved after 8 months. This treatment regimen can be advocated for similar nail dystrophies after excluding infective etiology.

(c) 2020 S. Karger AG, Basel

\section{Introduction}

Onychotillomania is an uncommon and misdiagnosed behavioral pattern focused on the nail apparatus. It is demarcated by the compulsive or irresistible urge in patients to constantly injure their own nails, with the fingers or tools, inflicting noticeable or even irreversible self-destruction of the nail unit. This urge may be conscious or unconscious $[1,2]$. Clinically, onychotillomania is characterized by a range of nonspecific findings, including bizarre morphology of the nail plate and damage to the nail bed and periungual skin. Clinical history is essential to making this diagnosis, as effective treatment modalities may focus on behavioral therapies and psychiatric medications [3]. Onychotillomania should be distinguished from onychophagia, which represents another form of self-induced damage of nails caused by nail biting [4].

Onychotillomania often poses a therapeutic challenge. It constitutes a persistent and difficult-to-manage problem, mostly because of its psychocutaneous nature as well as its high propensity to coexist with underlying neuropsychiatric illnesses or other behavioral disorders [2]. Although no large clinical trials have assessed the efficacy of treatments, cognitive-behavioral therapy, physical-barrier methods, and pharmacological treatments have shown some benefits in case reports. Onychotillomania remains a clinical and therapeutic challenge to dermatologists, pediatricians, internists, and psychiatrists in practice, as there are no evidence-based treatment methods [5].

\section{Case Report}

A 19-year-old, right-handed woman presented with a 4-year history of nail dystrophy affecting only the left thumbnail. On examination, the involved nail showed a typical lesion with nail plate thickening, discoloration, bizarrely shaped areas of nail cutting, and partial loss of its distal end. Exposed parts of nail bed were rough and scaly, while the periungual skin was inflamed with an

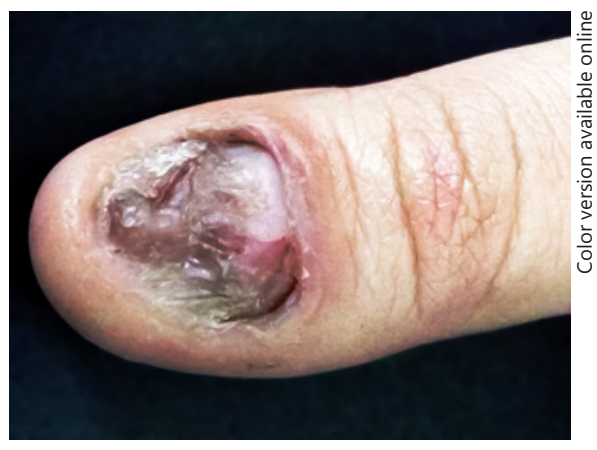

Fig. 1. Typical lesion of onychotillomania with nail plate thickening, discoloration, bizarrely shaped areas of nail cutting, and partial loss of its distal end. Exposed parts of the nail bed are rough and scaly, while the periungual skin is inflamed with a rough, ragged nail cuticle.

irregularly ragged nail cuticle (Fig. 1). The condition was resistant to repeated treatments with systemic and topical antifungals. She had no other nail, skin, or mucous membrane disorders and was generally in good physical health. She had suffered generalized anxiety disorder related to family and social troubles. After questioning, the patient endorsed that she was injuring/damaging her thumbnail during each anxiety episode. She used to pick and bite her nail as an automatic behavior and cut with sharp objects occasionally. Routine blood tests were within normal limits. Mycology workup was negative, and the patient refused nail biopsy. Nail psoriasis was excluded by history and dermatological examination of the patient and her relatives. Based on clinical history and classical nail changes, a diagnosis of onychotillomania was made.

The treatment plan was local steroid injection $(0.2 \mathrm{~mL}$ of $5 \mathrm{mg}$ / $\mathrm{mL}$ triamcinolone acetonide) monthly (for 3 months), then bimonthly, into the proximal nail fold that overlies the nail matrix. In addition, topical calcipotriol/betamethasone dipropionate combination was applied daily for 3 months and was then decreased to every other day. At the end of the third month, a noticeable improvement of the patient's nail was seen. The treatment was continued for 6 months, and complete clearance of nail changes and restoration of the nail cuticle and periungual tissues were observed after 8 months (Fig. 2). In parallel to treatment success, anxiety behavior diminished, and the patient started to spend less time picking her fingernail until she returned to normal, both physically and mentally. The 1-year follow-up revealed no recurrence of the condition.

\section{Discussion}

Onychotillomania is an under-recognized psychodermatosis that results from self-induced repeated trauma to the nail unit. The disorder presents with mild-to-severe typical, usually bizarre morphologies of the nail plate. The nail plate, nail bed, and periungual skin damage depends on the type of self-destructive behaviors employed 


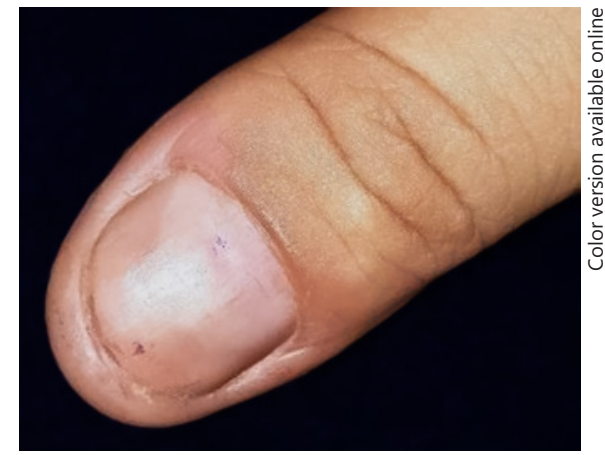

Fig. 2. Complete clearance of the nail changes after 8 months of treatment.

by the patient. The etiologic insult may be picking/rubbing the nail folds or using sharp instruments such as knives or razor blades $[3,4]$. Onychotillomania can involve fingernails and/or toenails. As in this case, the condition can affect a single nail, usually the thumbnail, it being the most accessible. In severe cases, it may lead to onychoatrophy due to irreversible scarring of the nail matrix. Very often, this occurs in psychologically normal children but may sometimes be associated with anxiety. In severe cases, onychotillomania may be a symptom of obsessive-compulsive disorders [6].

Based on clinical history, classical nail changes, and a 4 -year history of treatment failure the patient was diagnosed with onychotillomania. The diagnosis of onychotillomania is made clinically. Diagnosis can be a real challenge even for competent skin experts. Clinical features of the affected nails are nonspecific. To further complicate matters, both periungual and digit cutaneous tissues may be involved. This protean phenotype renders onychotillomania a dermatological masquerader able to mimic various nail disease entities $[2,3]$.

The overall average age of onset is 10.2 years, with a range of 6-18 years. There may be a slight female predominance. Most patients would use their fingers, mainly thumbs and index fingers, to pick at other nails, but using other instruments such as scissors, razor blades, and toothpicks can also occur [4]. The age of this patient coincides with the reported age range, and she used her fingers besides nail trimming instruments, sometimes intentionally, to induce the nail lesion.

Treating onychotillomania can also be challenging as no evidence-based treatment options are available. Frequent applications of distasteful topical preparations on the nail and periungual skin aiming to discourage patients from biting and chewing their fingernails [6] are employed. A common practice is occlusion of the nails from trauma by placing a barrier between the mouth and fingers; e.g., occlusive dressings such as gloves, bandages, and wrappings with adhesive tape [5]. This is applicable, to a large extent, to children but may be unsuitable for older patients.

The approach to this patient was to treat the marked nail dystrophy in a similar manner as in inflammatory nail disorders such as nail lichen planus and nail psoriasis. As an innovative approach, the combined local therapy, besides behavioral assistance, aimed to treat periungual inflamed tissue including the matrix to regrow a normal nail plate. Therefore, the previously mentioned treatment plan was used with an excellent response within few months. The rationale for calcipotriol use was that it acts as a vitamin $\mathrm{D}$ analogue, which regulates many physiological processes in the skin ranging from cellular proliferation, differentiation, and apoptosis to barrier maintenance and immune functions [7].

Both onychotillomania and the underlying psychosis could exacerbate each other, setting up a vicious cycle. In parallel to treatment success, anxiety behavior diminished, and the patient started to spend less time traumatizing her fingernail until she returned to normal both physically and mentally, breaking down the vicious cycle. Patients might become more motivated after seeing a clinical cure of their medical problem. The 1-year followup revealed no recurrence of the condition. In conclusion, the current case represents a complete resolution of the condition with local therapy, with complete restoration of the nail anatomy after 8 months. This treatment regimen can be advocated for similar nail dystrophies after excluding infective etiology.

\section{Statement of Ethics}

The study was approved by the Institutional Review Board of the Faculty of Medicine, Al Azhar University, and the patient has given her consent to publish photos and details of the case.

\section{Disclosure Statement}

The author has no conflicts of interest to disclose.

\section{Funding Sources}

The author did not receive any funding. 


\section{References}

1 Inglese M, Haley HR, Elewski BE. Onychotillomania: 2 case reports. Cutis. 2004 Mar; 73(3):171-4.

2 Sidiropoulou P, Sgouros D, Theodoropoulos K, Katoulis A, Rigopoulos D. Onychotillomania: A Chameleon-Like Disorder: Case Report and Review of Literature. Skin Appendage Disord. 2019 Feb;5(2):104-7.
3 Rieder EA, Tosti A. Onychotillomania: an underrecognized disorder. J Am Acad Dermatol. 2016 Dec;75(6):1245-50.

4 Grzesiak M, Pacan P, Reich A, Szepietowski JC. Onychotillomania in the course of depression: a case report. Acta Derm Venereol. 2014 Nov;94(6):745-6.

5 Halteh P, Scher RK, Lipner SR. Onychotillomania: diagnosis and Management. Am J Clin Dermatol. 2017 Dec;18(6):763-70.
6 Singal A, Daulatabad D. Nail tic disorders: manifestations, pathogenesis and management. Indian J Dermatol Venereol Leprol. 2017 Jan-Feb;83(1):19-26.

7 Umar M, Sastry KS, Al Ali F, Al-Khulaifi M, Wang E, Chouchane AI. Vitamin D and the Pathophysiology of Inflammatory Skin Diseases. Skin Pharmacol Physiol. 2018;31(2): 74-86. 